\title{
Politique
}

\section{Une prospective des relations France-Québec}

\section{Daniel Latouche}

Numéro 7, hiver 1985

Projection internationale du Québec

URI : https://id.erudit.org/iderudit/040479ar

DOI : https://doi.org/10.7202/040479ar

Aller au sommaire du numéro

Éditeur(s)

Société québécoise de science politique

ISSN

0711-608X (imprimé)

1918-6584 (numérique)

Découvrir la revue

Citer cet article

Latouche, D. (1985). Une prospective des relations France-Québec. Politique, (7), 67-86. https://doi.org/10.7202/040479ar d'utilisation que vous pouvez consulter en ligne.

https://apropos.erudit.org/fr/usagers/politique-dutilisation/ 


\title{
Débat
}

\section{Une prospective des relations France-Québec}

\author{
Daniel Latouche \\ Université McGill
}

Maintenant que les relations entre la France et le Québec ont réussi, finalement sans trop de difficultés mais probablement à la surprise générale, à prendre successivement les tournants historiques, culturels et économiques, on peut se demander si nous sommes arrivés à la fin des renversements. La ligne droite, tranquille et sans histoire, va-t-elle devenir la norme des relations entre nos deux communautés?

Qu'allons-nous faire de ces relations maintenant qu'elles ont été suffisamment tournées et retournées dans tous les sens qu'il faut déjà utiliser des historiens et des prospectivistes pour s'y retrouver. Se féliciter? Critiquer? Attendre?

* Conférence présentée le 17 mai 1984 lors de l'inauguration du Centre de coopération interuniversitaire franco-québécoise, à Paris. 
Mais plutôt que de penser à l'avenir, pourquoi ne pas se contenter de mieux organiser le présent. Il en est des relations comme des automobiles ou des maisons, elles ont toujours besoin d'un peu d'attention. Et surtout de ce genre d'attention qui ne fait pas la manchette, mais qui laisse des marques plus permanentes que les simples traces sur le papier trop recyclable des communiqués de presse.

Les thèmes que nous avons choisi d'aborder ne sauraient constituer des priorités, ils ne sont probablement même pas indispensables au maintien d'une coopération étroite entre la France et le Québec:

- le déblocage politique

- l'allègement bureaucratique

- le couronnement de l'excellence

- la conquête de marchés

- la compétition tranquille

- la relève de la garde

Disons d'abord un mot de l'esprit qui préside à cette réflexion. Les relations entre la France et le Québec demeurent pour l'instant une entreprise artificielle. Ce n'est pas faire injure au travail et à l'acharnement de ceux qui ont présidé à la mise en place de ces relations que de le reconnaître. Au contraire, la France et le Québec n'ont guère eu de difficulté à s'ignorer pendant deux siècles. Ils pourraient très bien continuer à le faire encore pendant longtemps. Le Québec demeure une terre d'Amérique, une ancienne colonie et une partie de l'espace politique, économique et social de l'Amérique du Nord. La géographie, l'histoire (la vraie, pas celle des discours) et la culture nous séparent. Dans bien des domaines nous sommes des étrangers les uns pour les autres.

Certes la langue nous rapproche. Mais elle nous éloigne aussi, car elle permet à nos différences d'émerger. Pouvoir découvrir 
ainsi en toute tranquilité tout ce qui nous sépare, et ensuite pouvoir l'exprimer à l'autre voilà bien le cachet particulier des relations France-Québec.

Ces vingt premières années de relations France-Québec auront été dominées par une sorte d'état de grâce - pour utiliser une expression qui, tant en France qu'au Québec, a déjà valeur de nostalgie - . À l'avenir, rien ne sera plus aussi simple. Certes on va pouvoir continuer à compter le nombre de stagiaires et de nouveaux accords sectoriels entre les deux sociétés. Mais cette comptabilité ne trompe déjà plus personne. Le temps des retrouvailes est terminé. Il va falloir inventer autre chose. Le défi est intéressant, surtout si on pense que depuis bon nombre d'années nous ne cessons de proclamer que nous sommes capables d'affronter de tels défis.

Cette francophonie, dont les relations France-Québec sont une composante essentielle, va-t-elle être autre chose que la matière première infiniment malléable des discours de ministres ou des rapports de colloques? Va-t-elle (enfin) devenir un espace de conflits et de concurrence, un lieu de pouvoir et d'excellence. Bref, quelque chose qui bouge et qui vit. N'est-il pas amplement temps de faire sortir les rapports France-Québec du simple cadre de la francophonie, et de faire passer cette dernière du culturel et de l'économique au politique. Cela me paraît être la condition essentielle pour que se mette en place à côté de l'espace géo-politique défini par les États-Unis et l'anglophonie un autre espace politique où le culturel davantage que l'économique joue un rôle structurant déterminant.

\section{Le déblocage politique}

Lorsqu'on examine d'un peu plus près les nombreux bilans et rapports sur la coopération France-Québec, on est frappé par l'absence de contenu politique dans cette multitude d'échanges, procès-verbaux, visites, communiqués de presse, «toasts» officiels. 
Dans le domaine des relations internationales, car c'est bien de cela qu'il s'agit lorsqu'on parle des relations France-Québec, c'est une situation passablement unique. Pourtant, s'il est un secteur qui en vu bien d'autres, c'est bien celui des relations inter-étatiques.

Certes, ce qu'il est convenu d'appeler l' «hypothèque canadienne» n'est jamais loin derrière, elle aura réussi à introduire une note politique dans les rapports France-Québec. C'est notre triangle bien à nous. Mais ce contenu politique qui vient automatiquement se surimposer sur toutes les relations France-Québec, n'est "politique» qu'à cause de la position particulière du Québec dans la fédération canadienne. Il est clair que pour la France et le Québec le "problème» canadien est quelque chose dont on se passerait bien. Que de réunions, de téléphones et de sous-entendus ont été investis dans cette question! Entre Québécois et Français, parler du Canada, ce n'est pas vraiment parler de politique, c'est plutôt parler de la température ou de ses rhumatismes (pour employer l'expression récente d'un ministre français). Il doit pourtant être possible à la France et au Québec de "parler politique» sans nécessairement parler d'Ottawa.

La restructuration des rapports politiques à l'échelle de la planète, le fait que des nouveaux rapports économiques viennent traverser ces relations politiques et les compliquer encore davantage crée une situation complètement nouvelle, qui force les relations France-Québec à prendre un nouveau départ. En 1964, on pouvait encore parler $d$ ' «invasion culturelle» américaine et de "culture française», cette dernière étant alors perçue comme résistant héroïquement à la première. Aujourd'hui l'invasion est terminée. L'envahisseur est venu et il est reparti. Il n'a pas imposé sa culture et sa langue - pas complètement tout au moins - mais il a laissé derrière lui ses catégories mentales, sa vision du progrès, sa notion de réseaux et surtout ses modes et ses outils de communication. De plus en plus ce sont les États-Unis qui définissent 
ce qu'est l'agenda planétaire. Leur politique est devenue notre politique.

Cette hégémonisation non pas tant dans le contenu que dans la forme est un fait de civilisation. Au Québec, nous sommes particulièrement bien placés pour voir tout ce qu'elle peut avoir de nocif. On a qu'à regarder la disparition culturelle et politique du Canada-Anglais, pour s'en convaincre! L'échec du projet canadien devrait nous faire réfléchir. Annonce-t-il l'impossibilité de construire au Canada un espace politique original qui témoignerait, d'une part, qu'il est possible d'être anglophone et de ne pas être américain et, d'autre part, qu'on peut être francophone dans ce continent anglophone d'Amérique. Aussi paradoxal que cela puisse paraître, cette interrogation est aussi vitale pour la France que pour le Québec.

Peut-il y avoir une coopération politique entre la France et le Québec? Il s'agit en fait de se demander si la politique a encore une place dans les relations entre les communautés nationales, ou si ces dernières ne sont plus que le résultat de déterminismes économiques dont les maîtres d'œuvre sont les firmes multinationales et les segments de marché. Bref, la régulation politique est-elle encore possible?

À regarder ce qui se passe dans les rapports est-ouest, nordsud, centre-périphérie, riches-pauvres, etc, on peut se permettre d'en douter.

Après quelques tentatives de concertation réunissant aussi bien tous les partenaires étatiques (ONU), que seulement les plus importants (sommets occidentaux), il semble qu'il faudra se résigner à imaginer de nouvelles approches pour gérer les rapports entre les communautés étatiques. De ce point de vue les relations entre la France et le Québec sont un bon exemple d'une façon de faire qui va peut-être contre l'«ordre naturel des choses diplomatiques», mais dont on doit aussi dire: «et pourtant elles existent». 
Sans tomber dans un messianisme ridicule, on doit cependant admettre que le caractère quelque peu exceptionnel des rapports entre une "province» de la fédération canadienne et un membre permanent du Conseil de sécurité constitue une voie de l'avenir que bon nombre d'acteurs impliqués dans des situations similaires auraient intérêt à suivre.

Cette volonté de faire accéder les rapports France-Québec à l'espace politique international ne se fera pas sans difficulté car elle menace bon nombre d'hégémonies bien installées dont la moindre n'est pas celle du monopole des États-nations sur la conduite des affaires internationales. Pourtant nous ne sommes plus à l'époque de l'Europe de Vienne et de l'Entente Cordiale. Les échanges d'ambassadeurs, les reconnaissances officielles ont encore leur importance, mais ils n'occupent plus tout l'avantscène. La notion même d'équilibre, pourtant si fondamentale dans les relations inter-étatiques, n'a plus aujourd'hui la même signification. Celle d'égalité, non plus. Il n'y a pas si longtemps encore on ne pouvait «déclarer la guerre» qu'à condition d'être membre officiel de la Communauté internationale.

Une évaluation détachée de la situation mondiale, en admettant que cela soit possible, ne peut que déboucher sur l'incertitude et l'ambiguïté. Sommes-nous, oui ou non, plus près aujourd'hui d'une catastrophe nucléaire qu'il y a 25 ans? Ou en sommes-nous simplement plus conscients? Les discours officiels des diplomates, et celui des contestataires, commencent eux-aussi à s'user. Est-ce mauvais signe? Qu'un ministre des Finances ou du Logement répète les mêmes choses pendant dix ans n'a jamais fait de mal à personne, sauf à l'imagination politique, mais n'estil pas dangereux de s'habituer au discours nucléaire? Allons-nous aborder le prochain millénaire avec un ordre du jour chargé des mêmes problèmes, des mêmes analyses et des mêmes façons de faire. La répétition semble être devenue le lot du politique et on 
a vu dans nos deux sociétés ce qu'il arrive de l'imagination lorsqu'elle vient au pouvoir. Peut-on encore avoir de l'imagination lorsque la réalité impose des choix à faire et des décisions à prendre.

Nous sommes malheureusement très loin des relations FranceQuébec. Il est probable que l'équilibre de la terreur ne sera jamais un sujet de débat très intense entre nos deux sociétés. Pourtant, c'est un exemple de ces nombreux points inscrits à l'agenda de l'humanité et sur lesquels il faudrait commencer à se parler. Se parler en français est une chose, se parler en français pour se répéter qu'il est agréable de se parler en français en est une autre.

Quel pourrait bien être l'objet de ces relations politiques entre la France et le Québec? Certainement pas la réforme de l'aménagement politique interne du Canada ou l'émergence du Québec sur la scène internationale. Dans un cas comme dans l'autre, ces deux questions ne se posent plus comme au milieu des années 60. «Parler politique» ne doit pas vouloir dire s'enfoncer un peu plus dans les méandres du triangle France-Québec-Canada.

Sur un plan strictement géographique, on peut penser que les États-Unis et le Canada constituent un terrain privilégié d'action commune. Sur un plan plus sectoriel, il est tout aussi évident que le domaine culturel et celui des communications représente le point d'entrée le plus indiqué pour une collaboration politique France-Québec.

Seule une analyse plus en profondeur de la «condition américaine» permettrait d'évaluer l'impact des changements qu'on peut déjà soupçonner et qui vont bientôt s'additionner pour créer dans ce pays un environnement fort turbulent. Parmi ceux-ci mentionnons-en quatre:

1. Le piétinement et finalement l'impasse devant lesquels se trouvent confrontées les exploitations de télévision "payante», «bi-directionnelle» et «conviviale». La raison en est simple: le 
contenu ne suit pas. De plus, la segmentation sans fin des marchés, et sur laquelle ces nouveaux industriels de la communication télévisuelle fondaient tous leurs espoirs, semble être en régression. Une nouvelle segmentation mêlant les critères démographiques et socio-économiques traditionnels avec des critères nouveaux (temps libre, degré d'intériorisation, ouverture culturelle) est en train d'émerger. Alors que la France est à mettre en place sa télévision payante, est-elle vraiment consciente de cette évolution?

2. Le problème démographique et culturel important que pose déjà la «latino-américanisation" des États-Unis. Le débat sur le bilinguisme y a déjà pris des allures qu'on connaît bien au Canada. Pour des raisons qui n'ont rien à voir avec la fierté culturelle, mais davantage avec des calculs de pouvoir politique et économique, le groupe hispanique refuse de plus en plus le modèle du «meltingpot». Dans une large mesure on se croirait revenu à cette époque où le groupe allemand, fort de son importance démographique, aurait pu demander une forme différente d'incorporation à la république américaine. Jusqu’à maintenant, seuls les signes les plus visibles... et les plus violents de cette présence latine se sont manifestés (ghettos, criminalité, ségrégation). L'impact culturel et idéologique n'est pas loin. Il va falloir l'évaluer.

3. La désaméricanisation de la «révolution informatique» est déjà commencée. Elle va se poursuivre. C'est la conséquence inévitable de son succès. Pour l'économie américaine les résultats risquent d'être encore plus désastreux que pour l'industrie des biens de consommation ou l'industrie automobile. Rien ne se déménage plus facilement qu'une «entreprise» de matière grise (pour ce qui est de son émergence, c'est une autre question!). Il n'y a pas de magie dans des phénomènes tels ceux de «Silicon Valley» et du «Research Triangle» de Caroline. 
4. Si les États-Unis demeurent toujours le principal exportateur de produits télévisuels (un phénomène dont on a trop tendance à ne voir que les conséquences hégémoniques), leur "contrôle» sur la création, la gestion et l'utilisation des banques de données et d'information n'est déjà plus ce qu'il était. L'imagination n'est pas un monopole américain et dans ce domaine, il suffit seulement d'un simple petit ajout pour créer une situation nouvelle. On peut même se demander si l'industrie de la communication va réagir de la même façon aux «lois" traditionnelles de la fabrication (coûts décroissants) et de la consommation (maturation des marchés).

On pourra trouver ce diagnostic trop optimiste (ou pessimiste). Mais l'intention n'est pas de prédire l'évolution d'un pays et d'un secteur qui ont fait de l'imprévisibilité leurs marques de commerce. Il s'agissait plutôt de montrer comment l'évolution de cette société et de ce secteur risque d'être déterminante pour toutes nos sociétés.

Le Québec possède aux États-Unis des «antennes» que la France n'a pas. Nos échanges commerciaux, scientifiques et culturels sont plus importants (ne parlons cependant pas de nos relations politiques). Sur ce pays nous avons quelque chose à dire. Il est donc temps que nos représentations politiques, nos fonctionnaires, nos décideurs économiques se parlent des États-Unis. C'est une étape nécessaire avant d'agir en commun sur le marché américain. À court terme, et dans un domaine plus universitaire, cela pourrait vouloir dire la création

1. d'un Institut franco-québécois sur les États-Unis (possiblement à Montréal) et en collaboration avec des institutions américaines.

2. d'un Forum sur les communications (réunissant les chercheurs, les «régulateurs» les industriels des deux sociétés).

Évidemment, c'est un peu curieux de suggérer que les ÉtatsUnis et l'Amérique du Nord devraient constituer un thème de 
réflexion France-Québec. S'il est un domaine où nos institutions universitaires et nos chercheurs ne sont plus dans la course, c'est bien celui des États-Unis et plus généralement celui du développement comparatif des sociétés occidentales. Il suffit de lire quelque peu les publications scientifiques dans ce secteur et de fréquenter les colloques internationaux pour remarquer l'absence quasi-universelle du français. Il y a d'autres domaines et d'autres terrains - l'Afrique, l'Amérique latine - où la France et le Québec pourraient envisager des actions communes, mais pour l'instant ce serait rêver que d'en parler...

Est-il besoin de préciser que pour le Québec, ce déploiement sur le monde des rapports France-Québec, surtout dans le domaine universitaire, est une condition essentielle pour que cette société puisse accéder à l'universel. Des raisons politiques et économiques qu'il serait trop déprimant de rappeler ici imposent une telle stratégie. Si le français se limite à être la langue des rapports France-Québec, on condamne nécessairement le Québec à une minorisation intellectuelle qui lui sera fatale et la France à un isolement, certes splendide, mais qui risque lui aussi de déboucher sur un avenir quelque peu rétréci.

S'il est assez facile de concevoir ce qu'une telle ouverture sur la politique peut comporter d'avantages pour le Québec (et le Canada), retrouve-t-on nécessairement la même comptabilité positive dans le cas de la France?

Notons tout d'abord qu'une telle ouverture n'enlève rien aux méthodes d'action et au positionnement plus traditionnels de la France. Il ne saurait être question de choisir entre le Conseil de sécurité, l'OCDE et le Québec. Cependant, le modèle des relations France-Québec s'inscrit parfaitement dans la logique d'une politique extérieure où la recherche de l'indépendance et la valorisation de certains principes occupent des places dominantes. En politique extérieure le «créneau» français passe nécessairement 
par la multiplication des expériences du type des rapports FranceQuébec. C'est la multiplication de ces situations «originales» qui seule pourra donner à la France une audience que son poids économique et militaire ne pourront seuls lui assurer.

De la même façon que le type et la qualité des rapports entre l'Allemagne et la France ont eu une importance déterminante pour la mise en place de l'Europe politique, dépassant ainsi largement le cercle immédiat des relations entre les deux pays, il n'est pas impensable d'imaginer que les rapports entre le Québec et la France pourraient débloquer sur des objectifs plus vastes.

Se parler de politique et même «faire de la politique» ensemble ne sera pas chose facile. Dans le cas du Québec, les marges de manœuvre sont limitées. Dans celui de la France, les responsabilités et les problèmes sont d'un autre ordre et atteignent des dimensions planétaires. À côté des États-Unis et du Canada, il y a aussi la communauté francophone internationale et celle des peuples «latins». Certes, pour l'instant, il faudra peut-être se contenter de réfléchir ensemble à ce que pourrait être une problématique commune et aux différentes formes que pourraient prendre un dialogue et une coopération politique France-Québec. Existe-t-il un espace politique où la France et le Québec peuvent se retrouver côte-àcôte et parfois même en situations de conflit?

\section{L'allégement bureaucratique}

Il s'agit là d'un thème fort populaire par les temps qui courent. Mais ce n'est pas pour suivre une mode que nous en parlons ici, mais davantage parce qu'il est toujours dangereux de laisser à une structure bureaucratique d'État le monopole d'un encadrement administratif. À plus forte raison lorsque cette structure a pris soin d'inclure dans son sigle le qualificatif de "permanente», comme c'est le cas de la Commission permanente franco-québécoise. 
Mentionnons tout de suite qu'il est impensable d'imaginer que cette coopération pourrait survivre très longtemps sans un encadrement administratif et politique dynamique. Il ne saurait donc être question de faire table rase. Parmi ces formules parallèles, on pourrait envisager:

- une décentralisation territoriale envers les municipalités et les régions des deux sociétés. Faut-il vraiment que tout soit décidé à Paris et à Québec?

- une décentralisation professionnelle (vers les groupes) afin que la coopération cesse d'être quelque chose qu'on reçoit de l'extérieur, présumément avec les budgets nécessaires, mais une activité qui s'inscrit dans la logique même des organisations.

- en France et au Québec les lieux pour organiser la concertation entre les principaux partenaires socio-économiques ne manquent pas. Il est temps d'impliquer ceux-ci plus activement dans des formules originales de partage des coûts et des décisions.

On objectera qu'il en résultera certainement un certain chaos. C'est probable. Il ne faut pas oublier que le Québec ne consacre actuellement qu'environ $\$ 10$ millions à ses programmes de coopération avec la France. Il s'agit finalement d'une somme minime (ce qui prouve que l'on peut faire beaucoup avec peu). À moins de décisions politiques importantes, il est peu probable que cette somme connaîtra une augmentation substantielle au cours des prochaines années. D'autres sources de financement, de nouveaux acteurs devront être trouvés. Les choses en seront plus compliquées. Des nouveaux enjeux feront leur apparition. Mais n'est-ce pas là le signe qu'une telle coopération est en bonne santé? 
On pourrait peut-être réfléchir quelque peu à la nécessité de continuer avec quelques-uns des piliers de la coopération FranceQuébec. Le premier de ces piliers, c'est la Commission permanente franco-québécoise. Dans les années 60 , c'était la mode d'établir ainsi de grandes commissions, sorte de directoires où tous les intérêts des parties en cause se trouvaient représentés afin de multiplier les possibilités de contacts. La lourdeur d'un tel mécanisme n'est plus à démontrer et le fait qu'on ait ajouté six Conseils d'orientation pour spécialiser les travaux de la Commission permanente vient de le confirmer. Nos fonctionnaires ont-ils encore besoin d'un tel mécanisme au sommet pour se parler et se concerter. Certes, ces rencontres annuelles sont devenues un rituel mais il est des folklores comme des scouts: «c'est bien mais à condition d'en sortir».

Les échanges de jeunes sont le deuxième joyaux de la couronne franco-québécoise. Les remettre en question sera sans doute considéré comme un crime de lèse-majesté - mais ce sont toujours les crimes les plus payants - L'Office franco-québécois pour la jeunesse est-il encore aussi indispensable qu'il y a 15 ans? $\mathrm{Ne}$ pourrait-on pas envisager que d'autres supports administratifs, décentralisés et professionnels, prennent la relève.

Et comme il n'est pas coutume de mettre un terme à une structure qui a fait ses preuves sans un baroud d'honneur, pourquoi la France et le Québec n'organiseraient-ils pas conjointement une conférence internationale pour faire le tour et dresser le bilan de ces programmes d'échanges de jeunes partout dans le monde. Et s'il faut un prétexte ou un anniversaire pour justifier un tel examen celui de l'Année internationale de la jeunesse est disponible.

Finalement il est grand temps de revoir un peu cette chasse gardée des missions et échanges entre nos deux communautés universitaires. Dans le secteur des sciences humaines, on compte sur les doigts de la main les livres et articles scientifiques écrits 
conjointement par des chercheurs français et québécois. On me dit que la situation est moins grise dans les sciences biologiques et physiques. Tant mieux. On se visite beaucoup mais nous n'en sommes plus à l'heure des émerveillements réciproques. Le temps des apprivoisements est terminé.

\section{Le couronnement de l'excellence}

Évidemment personne n'est contre l'excellence. Après quelques années de retard, cette qualité a rejoint la justice, la bonté et la compassion au Panthéon des qualités inattaquables. Mais ce cliché cache cependant une vérité. L'originalité, la différence, la créativité, la civilisation et l'indépendance, autant de caractéristiques que Français et Québécois aiment bien pratiquer - encore que dans le cas du Québec l'indépendance est en train de prendre une autre signification... - , toutes ces nobles vertus ne sont pas automatiquement des gages d'excellence. Ne pas être comme les autres parce qu'on parle français ne suffit pas.

C'est peut-être sur ce plan que la rupture entre le passé et cet avenir qu'on a tant de difficultés à voir émerger risque d'être la plus cruelie. Tant que nous avons vécu dans ce qu'il est convenu d'appeler la première génération de la civilisation de masse, il fut relativement facile pour nous, Québécois et Français, de se différencier et ainsi d'en appeler à un supplément d'originalité. Dans les deux cas, il a suffi d'être en retard sur ces grands bouleversements pour pouvoir prétendre communier à une civilisation supérieure. La France et le Québec, semblent avoir assez bien réussi à maintenir leur originalité réciproque. Jouissant du privilège du retard historique, ces deux sociétés ont finalement eu la tâche facile pour combler certains de leurs retards et se propulser au rang des sociétés développées et même post-développées. Le TGV et les CLSC sont les témoins de cette course à l'originalité. Ces 
deux innovations témoignent aussi que des réussites aussi quotidiennes que le transport et la santé peuvent être des signes de civilisation.

Multiplier les TGV et les CLSC, on le sait déjà, ne suffira plus. Si la «différence» demeure toujours une condition suffisante, elle n'est plus une condition nécessaire.

Cette recherche de l'excellence ne peut se faire dans l'intimité d'une relation à deux. Elle doit être connue de tous. Elle doit faire envie, faire des jaloux et forcer quelquefois les autres à l'imitation. Dans le domaine scientifique, il y a malheureusement belle lurette que «les autres» n'ont pas cherché à copier la production du monde francophone. De ce côté des choix s'imposent, et pour autant qu'ils soient véritables, ils seront difficiles et dangereux. Certes nos deux sociétés ne savent plus où donner de la tête afin de ne pas rater le virage technologique et scientifique. Nos gouvernements respectifs en ont fait des priorités nationales. Parfait! Qui oserait remettre en question un tel choix. Il y a bien quelques voix pour rappeler les coûts énormes de ces décisions, des voix qui à l'occasion descendent même dans la rue. Pourtant, bien peu d'attention a été consacrée à se demander si la notion même d'un tel virage technologique avait encore quelque sens.

L'exemple du Japon devrait pourtant nous forcer à réfléchir. En 1945, ce pays n'a pas décidé d'emprunter la voie que suivaient les États-Unis. Qui aurait pensé alors que la décision de ne pas se donner une industrie nationale d'aviation, une industrie nucléaire ou une industrie pharmaceutique aurait pu avoir des conséquences si positives. Et si l'exemple japonais ne suffit pas, il y a toujours celui du Canada avec ses réacteurs nucléaires les plus économiques, les plus fiables et les plus sécuritaires au monde, mais qui ne servent à rien et dont les retombées économiques sont inexistantes. Osera-t-on parler de l'expertise mondiale du Québec dans la 
construction de centrales hydroélectriques, expertise dont un seul pays pourrait avoir besoin. Malheureusement, il s'agit de l'URSS.

Ce long développement n'a d'autre but que de réhabiliter quelque peu les sciences humaines et sociales qui en ont passablement besoin par les temps qui courent. Voilà un domaine où l'excellence devrait nous venir naturellement - je dis «nous» pour parler de la communauté francophone - C'est un domaine où il n'est pas besoin de prendre un virage quelconque pour réussir, mais où pourtant la situation ne cesse de se détériorer. Nos quelques avantages comparatifs ne sont plus ce qu'ils étaient et nous ne pourrons plus vivre encore longtemps sur le capital accumulé à la suite de percées théoriques d'écoles parisiennes.

Il suffit de côtoyer quelque peu le monde de l'édition américaine, celui des grandes revues de sciences sociales, des centres de recherches, des colloques internationaux pour découvrir l'état pitoyable dans lequel baignent les sciences sociales francophones. Dans le domaine que je connais davantage, celui de la science politique, il faut parler de catastrophe. C'est tout de même significatif que nous ayons aussi peu à dire (sauf entre nous) sur le pouvoir et son exercice.

En sciences sociales, il nous faut l'équivalent d'un plan d'urgence. Rien de moins. Certes nous avons bon nombre d'intellectuels qui ont des choses fascinantes à dire sur l'évolution de la planète - occasionnellement, sur le dossier des relations FranceQuébec - , mais le travail d'intellectuel n'est pas celui de chercheur. Il nous faut au plus vite un réseau, des revues, des centres, de la compétition.

Ce centre de Jussieu n'est qu'un début. Disons même qu'il est un début prometteur. Il y a tant de domaines où Français et Québécois pourraient faire des choses ensemble qu'on hésite à les recenser. Au Québec, par exemple, il n'existe pas à proprement 
parler d'institut de recherches sur la France, ou sur les ÉtatsUnis. Rares sont nos étudiants qui se mettent à l'étude des langues étrangères. Nous n'avons pas d'instituts de recherches sur les sociétés occidentales.

Tout cela va coûter de l'argent, et les emplois créés seront peu nombreux. Alors qu'il est toujours possible d'importer la technologie et de prendre le virage plus facilement une fois que les autres ont reconnu le terrain, on ne peut en dire autant pour ce qui est de la compréhension de ces mécanismes mystérieux qui font que les sociétés existent et même disparaissent. De toute évidence, l'échange de quelques professeurs et les trop rares colloques où nous nous retrouvons aux mêmes tables ne suffiront pas. Un tel tourisme, aussi sympathique soit-il, est condamné à la marginalité. Au rythme d'un colloque par année, nous ne ferons que constater notre lente déchéance commune. Si c'est pour savoir si cela va plus mal en France qu'au Québec qu'on se réunit, alors mieux vaut passer à autre chose. L'objectif est simple - et je parle encore ici des sciences humaines et sociales - il faut que les chercheurs américains, britanniques, scandinaves et autres se bousculent aux portes pour publier leurs articles dans des revues francophones.

\section{La conquête de marchés}

Venons en au Canada. Évidemment, les Québécois n'aiment guère aborder ce sujet avec les Français. Ces derniers ont souvent appris à leurs dépens, qu'il est des sujets qu'il vaut mieux éviter. Pourtant, quel merveilleux «problème» à partager que le Canada. C'est peut-être le seul sur lequel nous avons une certaine exclusivité. Alors, pourquoi ne pas en profiter? D'ailleurs, il est probable que personne ne songe à nous le voler. 
Distinguons immédiatement le Canada de son gouvernement fédéral. Il faudrait même aller plus loin et distinguer l'État fédéral du parti qui en contrôle les destinées. Ce parti, en agissant avec un tel mépris à l'endroit du Québec, c'est tout le Canada qui s'en trouve déshonoré. Alors que le gouvernement d'Ottawa devrait être le premier à pousser le Québec à l'avant-scène internationale, il fait semblant que ce dernier n'existe pas. Non seulement le gouvernement fédéral choisit-il, en agissant de la sorte, de se couper un bras - et quand on vit à côté du géant américain, on a besoin de tous ses membres - mais il ne convainc personne à l'étranger, minant ainsi le peu de crédibilité qui pourrait lui rester.

Peut-on vraiment prendre le Canada au sérieux lorsqu'il parle du Liban, de l'Amérique centrale ou de la course aux armements alors que son aveuglement sur des choses qu'il devrait savoir est si évident.

Revenons au Canada. Il nous paraît évident que l'avenir des relations France-Québec passe par un règlement définitif de l'hypothèque canadienne. Il faut éviter qu'avec chaque changement de gouvernement, chaque élection ou chaque changement de leadership dans l'une ou l'autre de nos trois sociétés, tout soit remis en question. Cela exige trop d'efforts de la part de nos «spécialistes».

Mais comment fermer ce dossier? Certes, on ne peut pas faire semblant que le gouvernement canadien et le Canada n'existent pas. Pour la France, il y a là un marché potentiel trop important pour qu'on le passe sous silence. Et le Québec ne pourra empêcher pendant longtemps la France de s'y intéresser. Il ne reste plus qu'une solution: s'y intéresser ensemble. Pourquoi la France et le Québec ne tenteraient-ils pas d'y mener des actions économiques et culturelles conjointes. On pourrait même songer à un Institut franco-québécois d'études sur le Canada. 
Nous ne serons pas trop de deux pour conquérir un marché que les Canadiens eux-mêmes n'arrivent pas à préserver et que les Américains considèrent depuis longtemps comme le leur. Nos intérêts ne seront pas toujours complémentaires mais ils sont suffisamment importants pour qu'il soit possible de cheminer ensemble pendant un bon moment. Les micro-ordinateurs francoquébécois sont un excellent point de départ. Il va falloir les vendre à d'autres ces appareils. Pourquoi pas au Canada?

Il semble y avoir une tentation permanente pour la France de «laisser tomber» le Québec au profit d'une filière canadienne, tentation qui n'a d'équivalent que la «manie» québécoise de voir un complot derrière chaque éternuement français. Bien peu de gens à l'extérieur de la confrérie des spécialistes France-Québec comprennent quelque chose à ces transes existentielles. Il est facile de comprendre les susceptibilités québécoises en la matière. La seule façon d'en sortir est d'élargir le problème plutôt que de s'y enfoncer. Cela exigera une bonne dose de calme et de sérénité de la part des acteurs québécois qui devront se convaincre de passer par-dessus les manigances du gouvernement canadien.

Il faudra aussi passer à l'étape suivante. Pourquoi pas une rencontre tri-partite France-Québec-Canada? Avant de conclure immédiatement à l'habituel «Oui, mais ils ne voudront pas», il faudrait peut-être investir quelques énergies diplomatiques à fouiller la question. Il y va de l'intérêt des trois participants.

Le terrain semble actuellement propice pour de nouvelles initiatives. Et seul un tripartisme de bon aloi permettra de débloquer ce dossier.

\section{La compétition tranquille}

On dit qu'il y a pas mal de nuages au ciel des relations France-Québec. Il semble, par exemple, qu'il ne soit pas facile de prendre à deux le virage de l'électronique. On peut s'en plaindre. Il faut plutôt s'en féliciter. Enfin, nous avons un terrain où tous 
nos intérêts ne convergent pas, mais où l'économie générale d'une collaboration "malgré tout» est supérieure. C'est bon signe. Espérons que cela va continuer et même se répandre à d'autres secteurs.

Il va falloir s'habituer et apprendre à gérer cette compétition tranquille. Cela ne pourra se faire que si la France et le Québec développent ensemble des actions communes.

La relève de la garde

Tant en France qu'au Québec, l'effet combiné de la démographie du «baby-boom» et de la crise économique a entraîné de nombreux blocages. Au Québec, la génération des 35-45, confortablement assis sur ses fleurons de la révolution tranquille, ne laisse guère de place à la génération qui suit.

Si Français et Québécois veulent se parler, il leur faudra donner la parole à ceux qui ont quelque chose de nouveau et de différent à dire. L'émerveillement des retrouvailles ne touche guère cette relève. Cela ne fait que confirmer qu'on peut (enfin) passer à autre chose. 